\title{
A Hybrid Technique of Segmentation for Optimizing Features in Medical Images
}

\author{
Savita \\ Research Scholar \\ Sri Guru Granth Sahib \\ World University
}

\author{
Ada \\ Assistant Professor \\ Sri Guru Granth Sahib \\ World University
}

\begin{abstract}
Image segmentation is a part of digital image processing that deals to extract hidden patterns from the medical images. Medical image segmentation is a technique using to mean manually, fully or semi-automatically delineating the boundaries of tissue regions or an object. It is a process of changing the representation step of an image into some extent which is easy to analyze. In the recent research, cluster based, region based, threshold based and edge based approaches have been proposed. These approaches provide relational features from the image that provide various types of information. In medical science, image segmentation provides various achievements for decision making process. In the proposed work, image segmentation has been done using Hybrid PSO with multi scale super resolution approach. In the process of MSR, super resolution model has been implemented that computes gradient and sobel edges. After MSR, particle swarm optimization has been implemented that use fitness factor for computation of global fitness .After all the iterations and on the basis of global value, image has been segmented. Various parameters like PSNR, MSE, GCE, Random index, Variance and computation time have been measured for performance evaluation. The parameters show that proposed approach provides better results.
\end{abstract}

\section{Keywords}

Medical Image Segmentation, Multi scale resolution, PSO, PSNR, MSE

\section{INTRODUCTION}

To humans, an image is not just a random collection of pixels; it is a meaningful arrangement of regions and objects, a variety of images. Despite the large variations of these images, humans have no problem interpreting them. We can agree about the different regions in the images and recognize the different objects. Human visual grouping was studied extensively by the Gestalt psychologists in the early part of the 20th century (Wertheimer, 1938). They identified several factors that lead to human perceptual grouping: similarity, proximity, continuity, symmetry, parallelism, closure and familiarity. In computer vision, these factors have been used as guidelines for many grouping algorithms. The most studied version of grouping in computer vision is image segmentation. Image segmentation techniques can be classified into two broad families- (1) region-based, and (2) contour-based approaches. Region-based approaches try to find partitions of the image pixels into sets corresponding to coherent image properties such as brightness, color and texture. Contour-based approaches usually start with a first stage of edge detection, followed by a linking process that seeks to exploit curvilinear continuity. These two approaches need not be that different from each other. Boundaries of regions can be defined to be contours. If one enforces closure in a contour-based framework (Elder and Zucker, 1996;
Jacobs, 1996) then one can get regions from a contour-based approach. The difference is more one of emphasis and what grouping factor is coded more naturally in a given framework. A second dimension on which approaches can be compared is local vs. global. Early techniques, in both contour and region frameworks, made local decisions - in the contour framework this might be declaring an edge at a pixel with high gradient, in the region framework this might be making a merge/split decision based on a local, greedy strategy [9].

\section{REVIEW OF LITERATURE}

Banerjee, et al [1] "Bi level kaprus entropy based image segmentation using particle swarm optimization" In the field of Image Processing, Image segmentation is a low level but important task in entire image understanding system which divides an image into its multiple disjoint regions based on homogeneity. In most of the machine version and high level image understanding application, this is one of the important steps. Till date different techniques of image segmentation are available and hence there exists a huge survey literature in different approaches of image segmentation. Selection of image segmentation technique is highly problem specific. There is no versatile algorithm which is applicable for all kinds of images. Optimization based image segmentation is not explored much which can be applied to reduce complexity of the problem. The aim of the paper is to search for an optimized threshold value for Image Segmentation using Particle Swarm Optimization (PSO) algorithm where fitness function is designed based on entropy of the image.

En-Ui Lin et al [2] "Medical image segmentation using multi-scale and super-resolution method" In this paper we will use discrete wavelet transformation (DWT) and its enhanced version double density dual discrete tree wavelet transformations (D3-DWT) as they provide better spatial and spectral localization of image representation and have special importance to image processing applications, especially medical imaging. The multi-scale edge information from the sub-bands is then filtered through an iterative process to produce a map displaying extracted features and edges, which is then used to segment homogenous regions. We have applied our algorithm to challenging applications such as gray matter and white matter segmentations in Magnetic Resonance Imaging (MRI) images.

Castillo, A. et al [3] "Virtual Laboratory for Digital Image Processing" This paper introduces a virtual research center for the PC transforming of computerized imaging. This product application is intended to upgrade/remake high-determination pictures with a specific end goal to dissect the actualized calculations and also their optical gimmicks. The application is considered as a virtual research center for mechatronic, biomedical and electronic designing understudies. The introduced contextual investigations exhibit the exactness of the preparing chain utilized as a part of this virtual lab, and 
how the understudies could better comprehend points identified with remote sensing, PC vision, biomedical building, among others.

Coraor et al [4] "Processing in memory architectures for advanced picture handling" Continuing upgrades in semiconductor creation thickness are empowering new classes of framework on-a-chip architectures that join far reaching transforming rationale and high-thickness memory. A hefty portion of the capacities of these new architectures can be specially customized to the requests of ongoing computerized image processing. This paper assesses a few hopeful outlines, utilizing the criteria of picture handling execution, adaptability, manufacturability, and creation cost.

Deepak R. et al [5] "An Explicit Shape-Constrained MRFBased Contour Evolution Method for 2-D Medical Image Segmentation" Picture division is, all in all, a poorly postured issue and extra limitations need to be forced so as to accomplish the sought division result. While portioning organs in restorative pictures, which is the theme of this paper, a lot of former learning about the shape, appearance, and area of the organs is accessible that can be utilized to compel the arrangement space of the division issue. Among the different sorts of former data, the consolidation of earlier data about shape, specifically, is exceptionally difficult. In this paper, author display an unequivocal shape-compelled MAP-MRF-based form advancement technique for the division of organs in 2-D medicinal pictures. In particular, author speaks to the division shape unequivocally as a chain of control focuses. We then give the division issue a role as a shape advancement issue, wherein the development of the form is performed by iteratively illuminating a MAP-MRF marking issue. The advancement of the form is represented by three sorts of earlier data, to be specific: (i) appearance former, (ii) limit edgeness earlier, and (iii) shape former, each of which is consolidated as club possibilities into the MAPMRF issue. He utilize the expert slave double decay structure to settle the MAP-MRF marking issue in every cycle. In his investigations, he shows the application of the proposed system to the testing issue of heart division in non-difference processed tomography information.

Oprea, S. et al [6] "Computerized picture transforming connected in medications industry for location of broken headache medicine tablets" Interest in advanced picture preparing techniques comes from two main ranges: change of pictorial data for human understanding and handling of picture information for capacity, transmission, and representation for self-sufficient machine discernment. Today, there is no zone of specialized try that is not affected in same path by advanced picture handling. A significant range of imaging is in computerized visual examination of fabricated products. An average picture transforming assignment with items like this is to review them for missing or wrong parts. Recognizing a few oddities is a significant topic of modern review that incorporates a huge territory of items. The proposed paper introduces an application for identification of broken ibuprofen tablets utilizing picture handling methods. The application was actualized in an article situated picture transforming programming. The transforming plan can be adjusted for other functional applications from different spaces.

Prabala, A. et al [7] "Computerized yield cams improve picture transforming applications" Video cams intended for CCTV applications generally give a simple composite feature yield signal. In most advanced picture preparing applications, the simple feature yield from the cam is changed over into a computerized configuration by a simple to-advanced transformation process. In a customary CCD based cam, the CCD picture sensor creates an inspected simple sign which is prepared to make a composite simple feature yield. At the data of a picture preparing framework, the composite simple feature from the cam is digitized before any picture transforming is performed. In the event that the digitization is performed on the inspected simple yield of the CCD, inside the cam, then the two-stage information transformation procedure is supplanted by a solitary step process. Likewise, the balanced relationship between charge destinations on the CCD imager and the pixels in edge memory of the picture processor prompts more prominent accuracy amid picture handling. This has prompted the improvement of the Cohu 4110 arrangement of cams fusing an eight-bit computerized feature yield. This paper manages the profits of utilizing a digital yield cam and in addition a portion of the conceivable applications.

Thomas, G. et al [8] "Histogram Specification: A Fast and Flexible Method to Process Digital Images" Histogram detail has been effectively utilized as a part of advanced picture handling through the years. Principally utilized as a picture upgrade procedure, techniques, for example, histogram leveling (HE) can yield great stand out from pretty much no exertion as far as inputs to the calculation or the computational time needed. More expound histograms can tackle issues confronted by $\mathrm{HE}$ to the detriment of needing to characterize the last histograms in imaginative ways that may oblige some additional handling time yet are all things considered sufficiently quick to be considered for continuous applications. This paper proposes another method for defining a histogram to improve the picture contrast. To additional confirmation our confidence on histogram particular procedures, we likewise examine systems to change pictures, e.g., to help division approaches. Accordingly, as supporters of these systems, we might want to underscore the adaptability of this image transforming way to accomplish more than improving pictures.

Vajda, F. et al [9] "Systems and patterns in computerized picture transforming and PC vision" Summary structure just given. Advanced Image Processing is a quickly advancing field with developing applications in science and designing. The presentation addresses the essentials of significant subjects of digital image transforming and PC vision i.e. picture transforming and $\mathrm{PC}$ vision scientific classification, ordinary issues (counting their models and depictions), application zones, various leveled levels of picture preparing, some specimen picture handling calculations, control strategies for transforming (preprocessing, famous and typical preparing), essential systems for parallel handling (neighborhood operations, sub image/section parallel transforming and calculation/operational practical parallelism), application prerequisites continuously frameworks. The commonplace issues in picture transforming and PC vision applications are likewise portrayed.

Weiling Huang et al [10] "A novel street break discovery and recognizable proof technique utilizing computerized picture transforming strategies" Road split location is the base of roadway support and advanced picture preparing has been broadly utilized as a part of split recognition and ID. In this paper, a novel, compelling, proficient image processing system is proposed for the extraction of street splits from asphalt pictures. The principal stage is picture preprocessing which comprises of twofold division, morphological operations and evacuate calculation which uproots the confine 
dabs and range. Normally, after those operations above, numerous holes still exists in the break, the second stage proposed a novel calculation to interface those break breaks. It needs to judge the kind of the break on account of the distinction in distinctive sorts. The trial demonstrates that the surface break could be distinguished accurately and results demonstrated fulfilling and proficient.

\section{METHODOLOGY}

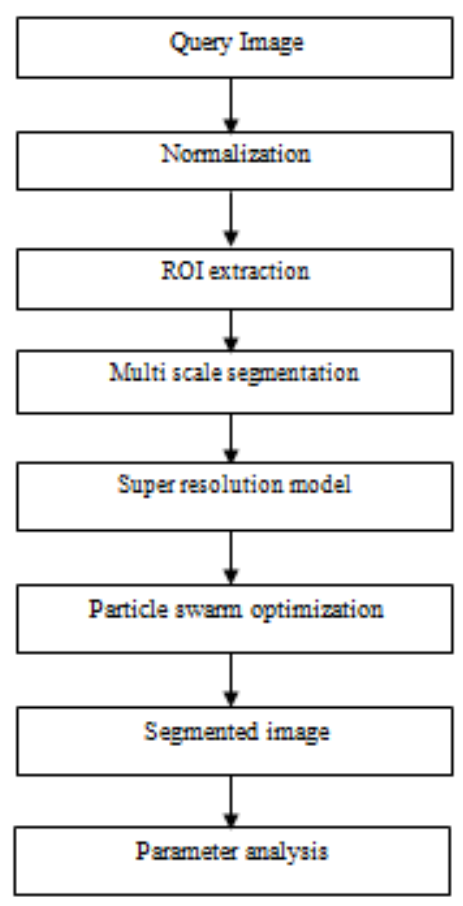

Fig 3.1. Flow of Work

Phase1: The query image has to be accused and normalized to that particular image. After the image accusation; the image has to be divided into different patterns.

Phase2: In the second phase, image has been divided into different regions for ROI extraction. After this process, FCM is implemented for multifractal analysis.

Phase3: After implementation of FCM approach, super resolution approach is used to reconstruct the high resolution images from low resolution images. After this, all the images are fused and optimized using PSO approach that identifies best solution for extraction of hidden features available in the medical image. And then the parameters like PSNR, MSE, GCE, Random index, Variance and Time are evaluated for performance evaluation.

\section{RESULTS}

\subsection{Parameters Used}

PSNR: PSNR stands for peak signal to noise ratio. The term peak signal-to-noise ratio (PSNR) is an expression for the ratio between the maximum possible value of a signal and the power of distorting noise that affects the quality of its representation. PSNR is usually expressed in terms of the logarithmic decimal scale. PSNR is used to measure the quality of image. The signal or input in this case is the original data, and the noise is the error introduced by compression. The PSNR is defined as:

$$
\begin{aligned}
& \text { PSNR }=10 \cdot \log _{10}\left(\frac{\mathrm{MAX}_{1}^{2}}{\mathrm{MSE}}\right) \\
&=20 \cdot \log _{10}\left(\frac{\mathrm{MAX}_{1}}{\sqrt{\mathrm{MSE}}}\right) \\
&=20 \cdot \log _{10}\left(\mathrm{MAX}_{1}\right)-10 \cdot \log _{10}(\mathrm{MSE})
\end{aligned}
$$

Although a higher PSNR generally indicates that the good quality of image. PSNR is most easily defined via the mean squared error (MSE). Here, $\mathrm{MAX}_{\mathrm{I}}$ is the maximum possible pixel value of the image. When the pixels are represented using 8 bits per sample, this is 255 . In this expression, PSNR is inversely proportional to the MSE, if the PSNR is high then MSE is low and if the PSNR is low then MSE is high.

Table 4.1 Comparison table of PSNR with various approaches

\begin{tabular}{|l|l|l|l|}
\hline Images & FCM & MSR & PSO \\
\hline Images 1 & 66.44496 & 70.16539 & 83.88146 \\
\hline Images 2 & 78.31663 & 68.57679 & 81.1924 \\
\hline Images 3 & 76.67548 & 66.09721 & 78.7335 \\
\hline Images 4 & 77.65446 & 69.44173 & 82.35146 \\
\hline Images 5 & 71.97241 & 69.40594 & 83.24213 \\
\hline
\end{tabular}

This table represents the value of PSNR using various segmentation approaches for image segmentation at different images.

PSNR

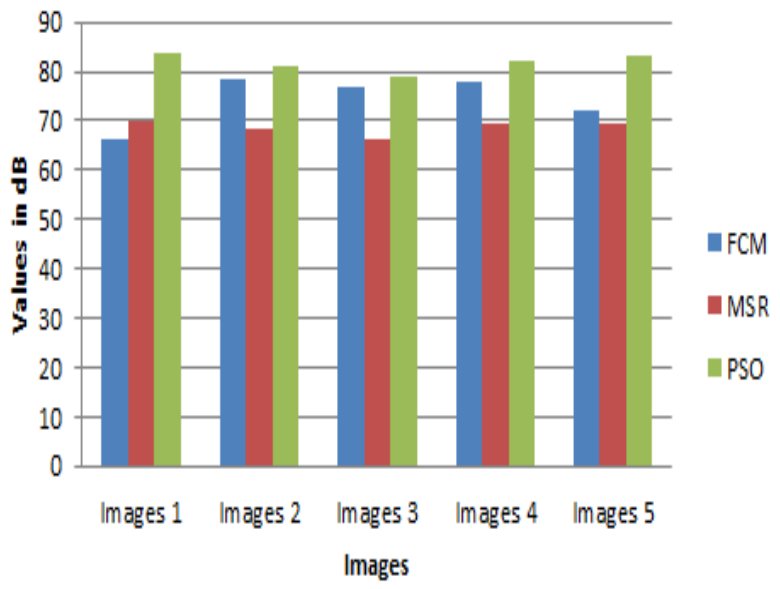

Fig 4.1 Comparative graph for various approaches using PSNR

Fig 4.1 represents graphical representation of performance evaluation of parameter PSNR with different approaches. As graph represents proposed work provides better PSNR than existing approach.

MEAN SQUARE ERROR: Mean square error (MSE) of an estimator measures the average of the squares of the "errors", that is, the difference between the estimator and what is estimated. If the value of MSE is low, then the quality of the image is better. In an analogy to standard deviation, taking the square root of MSE yields the root-mean-square error or root-mean-square deviation (RMSE or RMSD), which has the 
same units as the quantity being estimated; for an unbiased estimator.

The MSE is defined as:

$$
\text { MSE }=\frac{1}{m n} \sum_{i=0}^{m-1} \sum_{j=0}^{n-1}[I(i, j)-K(i, j)]^{2}
$$

Table 4.2 Comparison table of MSE with various approaches

\begin{tabular}{|l|l|l|l|}
\hline Images & FCM & MSR & PSO \\
\hline Images 1 & 10.39791 & 9.087658 & 4.159178 \\
\hline Images 2 & 7.493685 & 9.807657 & 5.668398 \\
\hline Images 3 & 7.700346 & 11.23086 & 7.52325 \\
\hline Images 4 & 7.572401 & 9.399367 & 4.960296 \\
\hline Images 5 & 8.562814 & 9.415467 & 4.476865 \\
\hline
\end{tabular}

This table represents the value of MSE using various segmentation approaches for image segmentation at different images.

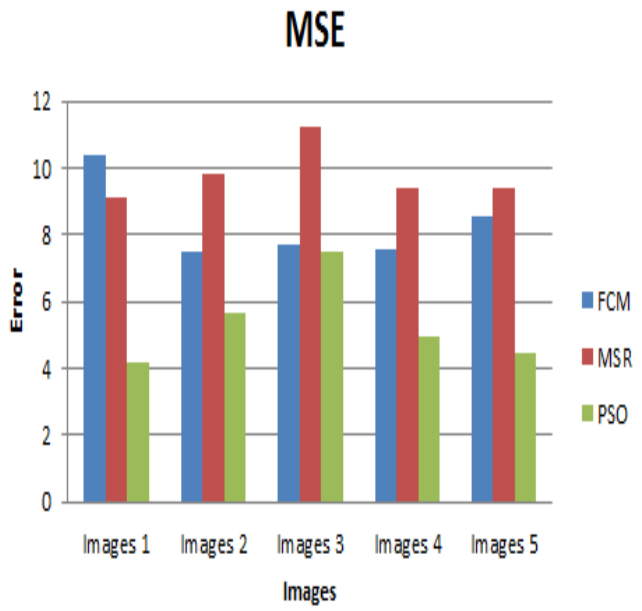

Fig 4.2 Comparative graph for various approaches using MSE

Fig 4.2 represents graphical representation of performance evaluation of parameter MSE with different approaches. As graph represents proposed work provides less MSE than existing approach.

RANDOM INDEX: The Random index (RI) between test and ground-truth segmentations $\mathrm{S}$ and $\mathrm{G}$ is given by the sum of the number of pairs of pixels that have the same label in $S$ and $G$ and those that have different labels in both segmentations, divided by the total number of pairs of pixels.

$$
\begin{aligned}
& \mathrm{RI} \quad=\frac{a+b}{a+b+c+d}\left[\left(\frac{n}{2}\right)\left[0.5 \sum_{i}\left(\sum_{j} n i j\right)^{2}+\sum_{j}\left(\sum_{i} n i j\right)^{2}\right]-\right. \\
& \left.\left.\sum \sum n i j\right)^{2}\right] / /\left(\frac{n}{2}\right) \quad \text { where, }
\end{aligned}
$$

a, the number of pairs of elements in $S$ that are in the same set in $\mathrm{U}$ and in the same set in $\mathrm{V}$; $b$, the number of pairs of elements in $\mathrm{S}$ that are in different sets in $\mathrm{U}$ and in different sets in V; c, the number of pairs of elements in $S$ that are in the same set in $\mathrm{U}$ and in different sets in $\mathrm{V}$; $\mathrm{d}$, the number of pairs of elements in $\mathrm{S}$ that are in different sets in $\mathrm{U}$ and in the same set in $\mathrm{V}$.
Table 4.3 Comparison table of Random Index with various approaches

\begin{tabular}{|l|l|l|l|}
\hline Images & FCM & MSR & PSO \\
\hline Images 1 & 0.510687 & 0.519282 & 0.829533 \\
\hline Images 2 & 0.56104 & 0.579862 & 0.880323 \\
\hline Images 3 & 0.546379 & 0.567714 & 0.866379 \\
\hline Images 4 & 0.509194 & 0.526931 & 0.827561 \\
\hline Images 5 & 0.572334 & 0.563402 & 0.892334 \\
\hline
\end{tabular}

This table represents the value of Random Index using various segmentation approaches for image segmentation at different images.

\section{Randon Index}

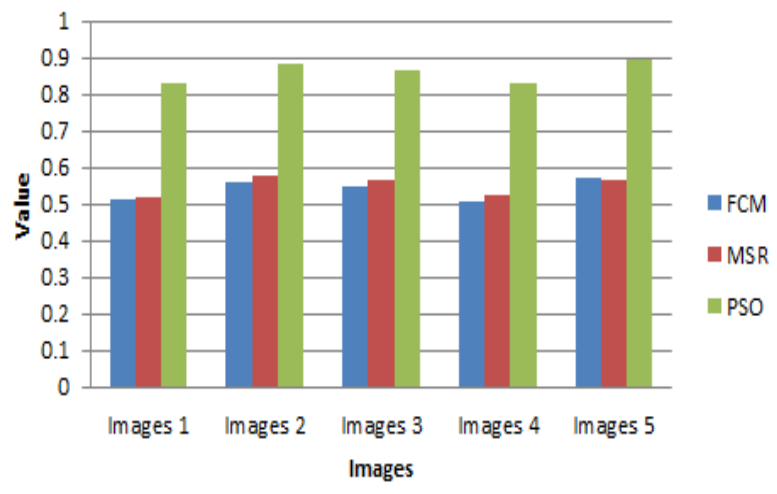

Fig 4.3 Comparative graph for various approaches using Random Index

Fig 4.3 represents graphical representation of performance evaluation of parameter Random Index with different approaches. As graph represents proposed work provides better Random Index than existing approach.

\section{GLOBAL CONSISTENCY ERROR:}

The Global Consistency Error (GCE) measures the extent to which segmentation can be viewed as a refinement of the other. Segmentations which are related are considered to be consistent, since they could represent the same image segmented at different scales. The formula for GCE is as follows,

$$
\mathrm{GCE}=\frac{1}{n} \operatorname{Min} \sum_{i} E(S 1, S 2, P i), \sum_{i} E(S 2, S 1, P i)
$$

where, segmentation error measure takes two segmentations $\mathrm{S} 1$ and $\mathrm{S} 2$ as input, and produces a real valued output in the range [0::1] where zero signifies no error. Global Consistency Error (GCE) forces all local refinements to be in the same direction.

Table 4.4 Comparison table of Global Consistency Error with various approaches

\begin{tabular}{|l|c|c|c|}
\hline Images & FCM & MSR & PSO \\
\hline Images 1 & 0.633139 & 0.043326 & 0.01 \\
\hline Images 2 & 0.57901 & 0.024294 & 0.01 \\
\hline Images 3 & 0.253228 & 0.028917 & 0.01 \\
\hline Images 4 & 0.924313 & 0.045743 & 0.01 \\
\hline Images 5 & 0.152375 & 0.051999 & 0.01 \\
\hline
\end{tabular}

This table represents the value of GCE using various segmentation approaches for image segmentation at different images. 


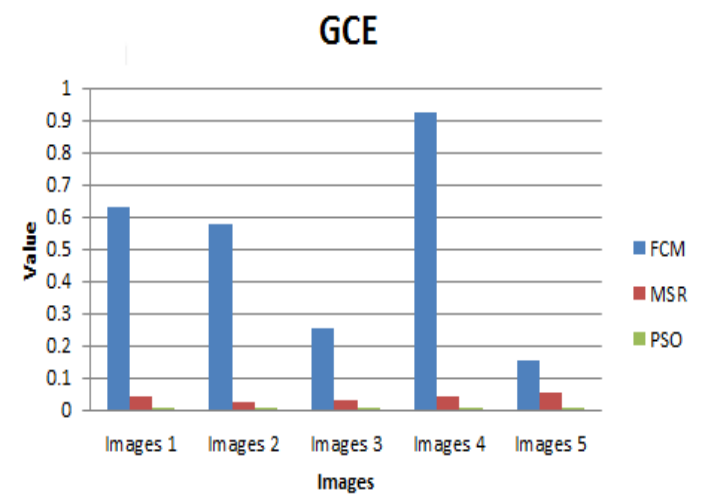

Fig 4.4 Comparative graph for various approaches using GCE

Fig 4.4 represents graphical representation of performance evaluation of parameter GCE with different approaches. As graph represents proposed work provides less GCE than existing approach.

VARIANCE: The Variation of Information (VoI) metric defines the distance between two segmentations as the average conditional entropy of a segmentation given the other, and thus roughly measures the amount of randomness in a segmentation which cannot be explained by the other. Lower the $\mathrm{VoI}$ value better is the result. $\mathrm{VoI}$ is defined as:

$$
\mathrm{VoI}\left(\mathrm{C}, \mathrm{C}^{\prime}\right)=\mathrm{H}(\mathrm{c})+\mathrm{H}\left(\mathrm{C}^{\prime}\right)-2 \mathrm{I}\left(\mathrm{C}, \mathrm{C}^{\prime}\right)
$$

The proposed measure, termed the Variation of Information (VoI), is a metric and is related to the conditional entropies between the class label distributions of the segmentations. The measure has several promising properties but its potential for evaluating results on natural images where there is more than one ground-truth clustering is unclear.

Table 4.5 Comparison table of Variance with various approaches

\begin{tabular}{|l|c|c|c|}
\hline Images & FCM & MSR & PSO \\
\hline Images 1 & 4.913863 & 4.853572 & 4.715561 \\
\hline Images 2 & 4.342918 & 4.184555 & 4.143981 \\
\hline Images 3 & 4.5794 & 4.432484 & 4.3794 \\
\hline Images 4 & 4.854181 & 4.801285 & 4.656603 \\
\hline Images 5 & 5.208183 & 5.204958 & 5.008183 \\
\hline
\end{tabular}

This table represents the value of Variance using various segmentation approaches for image segmentation at different images.

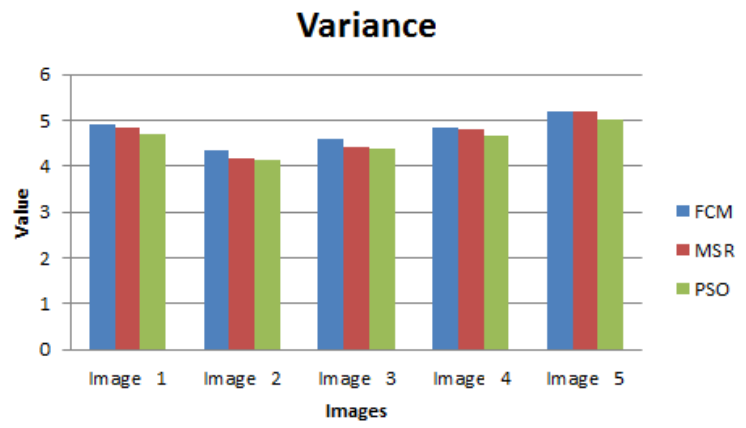

Fig 4.5 Comparative graph for various approaches using Variance
Fig 4.5 represents graphical representation of performance evaluation of parameter Variance with different approaches. As graph represents proposed work provides less Variance than existing approach.

TIME: This measure represents the value of execution time taken by various segmentation approaches for image segmentation at different images.

Table 4.6 Comparison table of Time Taken with various approaches

\begin{tabular}{|l|l|l|l|}
\hline Images & FCM & MSR & PSO \\
\hline Image 1 & 3.327213 & 3.108121 & 0.033204 \\
\hline Image 2 & 2.962562 & 3.147775 & 0.034141 \\
\hline Image 3 & 3.440747 & 3.120369 & 0.033906 \\
\hline Image 4 & 3.084631 & 3.114323 & 0.033812 \\
\hline Image 5 & 5.579126 & 3.236361 & 0.03405 \\
\hline
\end{tabular}

This table represents the value of Time using various segmentation approaches for image segmentation at different images.

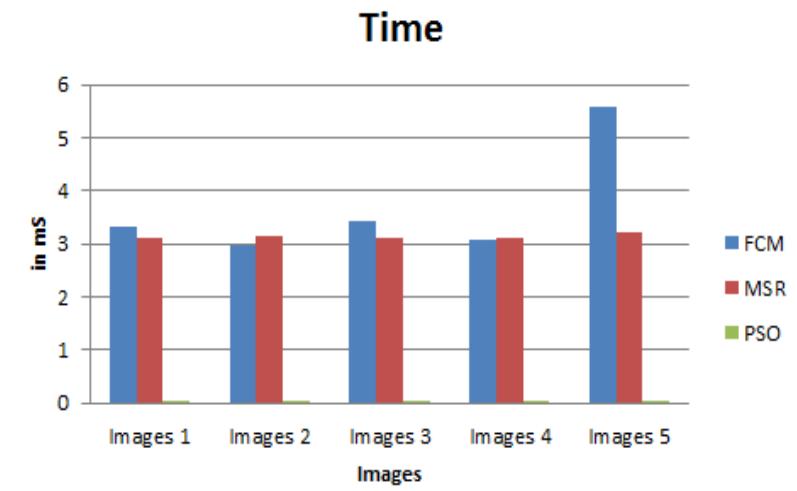

Fig 4.6 Comparative graph for various approaches using time

Fig 4.6 represents graphical representation of performance evaluation of parameter Time with different approaches. As graph represents proposed work takes less Time than existing approach.

\section{CONCLUSION \& FUTURE SCOPE 5.1 Conclusion}

Image processing is the field of processing the images by applying mathematical formulas. Now a day's medical images or video frames are used in the form of signal processing and these images are further given as input, the output may be an image or different parameters related to the images. A good segmentation is typically one in which pixels in the same category have similar grayscale of multivariate values and form a connected region, neighboring pixels which are in different categories have dissimilar values. It is typically used to locate objects and boundaries (lines, curves, etc.) in images.

- The proper Region of Interest has been detected.

- FCM approach has been implemented for multifractal analysis.

- All the images have been segmented by using different computation of the super resolution model that computes values of gradient and edges available in the images. 
- The images have been fused and optimized using PSO approach that identifies best solution for extraction of hidden features available in the medical image. In PSO image, features have been computed and fitness value has been evaluated after single iteration. From entire iterations global fitness has been computed that has been used for segmentation of desired image.

- Various parameters (PSNR, MSE, GCE, Time, Variance, Random Index) have been computed for performance evaluation of proposed work. On the basis of these parameters, one can conclude that proposed work provide better results than previous approaches.

\subsection{Future Scope}

- In future, the image optimization can be done to improve the results and make them efficient. Image optimiz8ation can be done by applying many other optimization algorithms.

- Other approaches can be developed that can be used for finding of ROI in the input image. Automatic detection of area can solve various problems of image segmentation. The optimization algorithms such as ant colony, bacterial foraging algorithm etc can be implemented.

\section{REFERENCES}

[1] Banerjee "Bi level kaprus entropy based image segmentation using particle swarm optimization", Computer Communication, Control and Information Technology (C3IT), pp 1-4, 2015.

[2] En-Ui Lin, Michael McLaughlin “ Medical Image Segmentation using Multi-Scale and Super-Resolution Method" , IEEE Conf. on MIS, pp 34-44, 2014.

[3] Castillo, A., Ortegon, J. "Virtual Laboratory for Digital Image Processing",Latin America Transactions, vol. 12, pp. 1176 - 1181, 2014.

[4] Coraor, L., Landis, D., Hulina, P. "Computing in memory architectures for digital image processing",
International Workshop on Memory Technology, Design and Testing, pp. $8-15,2014$.

[5] Deepak R. Chittajallu “An Explicit Shape Constrained MRF-Based Contour Evolution Method for 2-D Medical Image Segmentation”, IEEE, 2013.

[6] Oprea, S. "Computerized picture transforming connected in medications industry for location of broken headache medicine tablets", IEEE Conf. on Medicine Tablets, pp 345-355, 2010.

[7] Prabala, A. "Digital output cameras enhance image processing applications", WESCON/'93. Conference Record, pp 120-124, 2013.

[8] Thomas, G., Flores-Tapia, D., Pistorius, S. "Histogram Specification: A Fast and Flexible Method to Process Digital Images", IEEE Transactions on Instrumentation and Measurement, vol. 60, pp. 1565 - 1578, 2012.

[9] Vajda, F. "Systems and patterns in computerized picture transforming and PC vision", IEEE conf. on PC vision, pp 2-15, 2012.

[10] Weiling Huang, Ning Zhang "A novel road crack detection and identification method using digital image processing techniques", 7th International Conference on Computing and Convergence Technology (ICCCT), pp. $397-400,2013$.

[11] Gourav, Singh, S.R. "Enhancement of contrast and resolution of gray scale and color images by wavelet decomposition and histogram shaping and shifting" IEEE International Conference on Medical Imaging, m-Health and Emerging Communication Systems, pp. 300-305, 2014.

[12] Shihab A. Hameed "Hybrid and Multilevel Segmentation Technique for Medical Images", IEEE, 2013.

[13] Hasikin, K, Isa, N.A.M. "Enhancement of the Low Contrast Image Using Fuzzy Set Theory" IEEE 14th International Conference on Computer Modelling and Simulation, pp. 371-376, 2012. 